\title{
Otimização do solvente para extração de compostos antioxidantes da polpa de abacate utilizando o planejamento de misturas simplex-centroide
}

\author{
Tahís Regina Baú, Carla Rosane Barboza Mendonça
}

https://doi.org/10.4322/mp.978-65-991393-3-8.c2

\section{Resumo}

O abacate é uma fruta tropical com elevado valor nutritivo e fonte de importantes substâncias bioativas, como os compostos fenólicos e antioxidantes. A extração de compostos fenólicos e antioxidantes em alimentos é difícil, devido às diferentes características destes compostos e a complexidade das matrizes alimentares. Objetivou-se com este estudo otimizar as condições de extração de compostos fenólicos e antioxidantes da polpa de abacate da variedade Quintal por meio da aplicação de um planejamento de misturas simplex-centróide. Os compostos fenólicos e antioxidantes foram extraídos da polpa de abacate utilizando como solventes água, acetona, etanol e metanol, em diferentes combinações, totalizando 16 ensaios, conforme o planejamento experimental empregado. As misturas foram analisadas em relação ao teor de compostos fenólicos pelo método de Folin-Ciocalteau e atividade antioxidante pelos métodos ABTS e DPPH. De acordo com a análise de superfície de resposta e desejabilidade, a combinação de 50\% de água e 50\% de acetona revelouse a mais apropriada para a extração de compostos fenólicos e antioxidantes da polpa de abacate variedade Quintal.

Palavras-chave: ABTS, compostos bioativos, DPPH, metodologia de superfície de resposta, solventes, variedade Quintal.

\section{Introdução}

O abacate (Persea americana Mill.) é uma importante fruta tropical comercial (WANG; BOSTIC; GU, 2010), possui forma de drupa com casca delgada, de coloração verde oliva-brilhante, que apresenta elevado teor de água, em torno de $71,51 \%$ e óleo de alta qualidade (SAMPAIO; LEONEL, 2008). O abacate é fonte de ácidos graxos insaturados, fibras, potássio, vitamina B (GOMEZ-LOPEZ, 1998) e compostos lipofílicos bioativos, como a vitamina $E$, carotenoides, esteróis e outros, que tem demonstrado atividade antioxidante (LEE; KOO; MIN, 2004). Segundo a American Diet Associati on (ADA) o abacate pode ser classificado como um alimento funcional, devido ao seu alto valor nutritivo e comprovados efeitos benéficos para a saúde humana (ADA, 1999).

Vários compostos antioxidantes naturais já foram isolados a partir de diferentes tipos de materiais de plantas, tais como oleaginosas, cereais, legumes, frutas, folhas, 
raízes, especiarias e ervas (RAMARATHNAM et al., 1995). O abacate é uma importante fonte de compostos bioativos, que protegem as células humanas contra os efeitos prejudiciais dos radicais livres. Além disso, os compostos fenólicos são conhecidos por apresentar outras propriedades benéficas à saúde, como atividade antioxidante, efeito anti-inflamatório, antitumoral e antimicrobiano (LUTHRIA, 2008). Os principais antioxidantes presentes na polpa do abacate são os carotenoides (xantofilas, principalmente a luteína) bem como as vitaminas C e E (JACOBOVELÁSQUEZ; HERNÁNDEZ-BRENES, 2012; LU et al., 2005). Além disso, a semente do abacate é rica em compostos fenólicos, como a catequina e epicatequina, entre outros (GEISSMAN; DITTMAR, 1965). A capacidade antioxidante de vegetais, como 0 abacate, pode ser afetada por diversos fatores, como as condições de cultivo, variedade, manipulação pós-colheita e estádio de maturação dos frutos (KEVERS et al., 2007).

A extração de compostos fenólicos e antioxidantes das amostras alimentícias é um processo complexo e desafiador, já que mais de 800 compostos foram isolados de fontes naturais (LUTHRIA, 2006; ROBBINS, 2003). Esses compostos mostram estrutura química variada e podem estar conjugados com diferentes grupos (LUTHRIA, 2008), o que influencia substancialmente a sua polaridade, tornando difícil o desenvolvimento de um método para extração de todos os compostos desta natureza presentes em diferentes matrizes alimentares. Fatores como a composição e polaridade da solução extratora, tempo de extração, temperatura, $\mathrm{pH}$, relação sólido:líquido e tamanho da partícula podem influenciar a eficiência da extração de compostos fenólicos e antioxidantes em frutas (POMPEU; SILVA; ROGEZ, 2009).

A extração de compostos fenólicos e antioxidantes é uma importante ferramenta para recuperação e purificação desses compostos bioativos. A solubilidade dos compostos fenólicos e antioxidantes é governada pela polaridade do solvente extrator utilizado, o que pode influenciar significativamente na eficiência da extração, refletindo na maior ou menor recuperação destes compostos. Neste sentido, é necessário que ocorra uma seleção adequada de solventes para a extração, e um estudo para a otimização da extração destes compostos em cada matriz alimentar, utilizando o tipo e a proporção mais adequada de solventes.

$\mathrm{Na}$ literatura existe uma quantidade considerável de trabalhos que avaliam teores de compostos fenólicos e antioxidantes em alimentos, porém, não há uma padronização das condições de extração específicas para cada matriz alimentar. Dessa forma, os resultados obtidos tornam-se difíceis de comparar, pois apenas o conteúdo final extraído de compostos fenólicos e antioxidantes é quantificado (SPIGNO; TRAMELLI; FAVERI, 2007). O processo de extração deve fornecer o máximo rendimento das substâncias de interesse, assim sendo, objetivou-se neste estudo otimizar as condições de extração de compostos fenólicos e antioxidantes da polpa de abacate da variedade Quintal por meio da aplicação de um planejamento simplex-centróide.

\section{Material e Métodos}

\subsection{Material}

Os abacates da variedade Quintal foram obtidos no comércio local da cidade de Francisco Beltrão/PR. Foram selecionados frutos maduros, com adequada firmeza da polpa, teor de sólidos solúveis de 7 a $8{ }^{\circ}$ Brix, sendo descartados os que apresentassem injúrias físicas ou contaminação visível. 


\subsection{Métodos}

2.2.1 Planejamento de misturas do tipo simplex-centroide para escolha da mistura de solventes com maior poder extrator dos compostos fenólicos e antioxidantes

Para otimizar a extração de compostos fenólicos e antioxidantes do abacate foi utilizado o planejamento experimental para misturas do tipo simplex-centroide (SCHEFFÉ, 1963) com quatro componentes de diferentes solventes extratores $\left(X_{1}=\right.$ água, $X_{2}=$ acetona, $X_{3}=$ etanol e $X_{4}=$ metanol) totalizando 16 ensaios (Figura 1 ). $O$ planejamento experimental do tipo simplex-centroide, originalmente descrito por Scheffé (1963) é um planejamento para misturas que pode ser representado por um triângulo, para três componentes ou um tetraedro para quatro componentes. Para explorar as condições de mistura do espaço inteiro, as misturas usadas são os pontos situados nos vértices e no meio das arestas, no qual cada ponto representa uma combinação dos componentes da mistura. Os pontos 1, 2, 3 e 4 (vértices do triângulo) corresponderam aos solventes água, acetona, etanol e metanol, respectivamente. Os pontos 5 a 10 são as misturas binárias de dois solventes. Os pontos 11 a 14 são as misturas ternárias e os pontos 15 e 16 são as misturas quaternárias dos solventes.

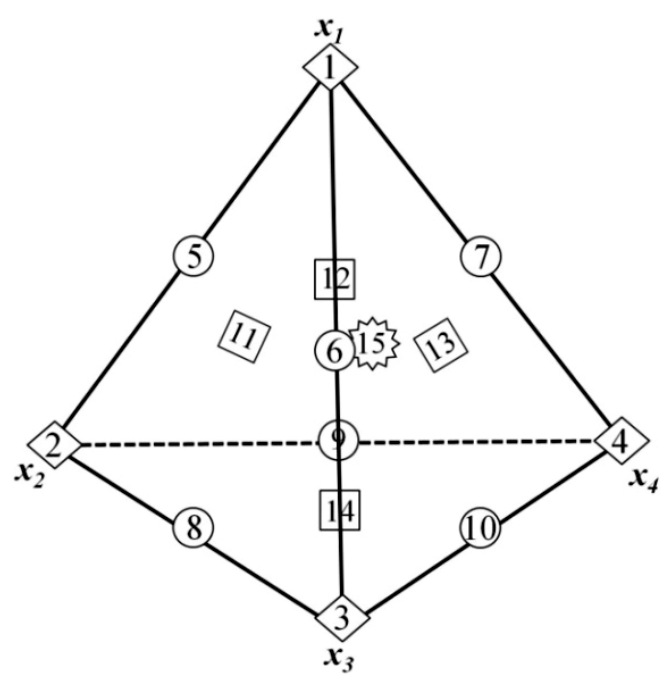

\begin{tabular}{lll} 
& $\mathrm{E}$ & $\left(x_{1}: x_{2}: x_{3}: x_{4}\right)$ \\
\hline Solventes puros & 1 & $1: 0: 0: 0$ \\
& 2 & $0: 1: 0: 0$ \\
& 3 & $0: 0: 1: 0$ \\
& 4 & $0: 0: 0: 1$ \\
\hline Misturas binárias & 5 & $0.50: 0.50: 0: 0$ \\
& 6 & $0.50: 0: 0.50: 0$ \\
& 7 & $0.50: 0: 0: 0.50$ \\
& 8 & $0: 0.50: 0.50: 0$ \\
& 9 & $0: 0.50: 0: 0.50$ \\
& 10 & $0: 0: 0.50: 0.50$ \\
\hline$\square$ Misturas Ternárias & 11 & $0.33: 0.33: 0.33: 0$ \\
& 12 & $0.33: 0.33: 0: 0.33$ \\
& 13 & $0.33: 0: 0.33: 0.33$ \\
& 14 & $0: 0.33: 0.33: 0.33$ \\
\hline m. Mistura Quaternária & 15 & $0.25: 0.25: 0.25: 0.25$ \\
\hline
\end{tabular}

Figura 1. Planejamento experimental simplex-centroide com 4 componentes. Fonte: SCHEFFÉ, 1963.

\subsubsection{Extração e quantificação dos compostos fenólicos e antioxidantes}

Foram pesados cerca de $3 \mathrm{~g}$ de amostra de abacate in natura e adicionados $8 \mathrm{~mL}$ de solvente extrator, conforme planejamento experimental (Figura 1). A extração dos compostos fenólicos e antioxidantes foi realizada conforme metodologia descrita por Pereira et al. (2013), com modificações. As amostras foram agitadas em shaker por 1 h a $150 \mathrm{rpm}$, seguidas de centrifugação a $3940 \mathrm{~g} / 15 \mathrm{~min}$ a $4{ }^{\circ} \mathrm{C}$. O sobrenadante foi coletado e submetido às determinações de compostos fenólicos e atividade antioxidante.

Os compostos fenólicos foram quantificados de acordo com o método de FolinCiocalteau descrito por Swain e Hillis (1959), utilizando curva padrão de ácido gálico 
$\left(0,02\right.$ a $\left.0,07 \mathrm{mg} \cdot \mathrm{mL}^{-1}\right)$ e o resultado expresso como $\mathrm{mg}$ equivalentes de ácido gálico por $100 \mathrm{~g}$ de amostra (mg EAG.100g $\left.{ }^{-1}\right)$.

A atividade antioxidante dos diferentes extratos foi medida pela capacidade do composto avaliado em doar íons hidrogênio ao radical livre 2,2-difenil-1-picrilhidrazil $(\mathrm{DPPH} \bullet$ ) segundo o método descrito por Brand-Williams, Cuvelier e Berset (1995). Foi utilizada uma curva padrão de Trolox (10 a $1000 \mu \mathrm{mol}^{-L^{-1}}$ ) e os resultados foram expressos em $\mu \mathrm{mol}$ Trolox. $\mathrm{g}^{-1}$ de amostra.

A atividade antioxidante também foi avaliada pelo seqüestro do cátion radical $\mathrm{ABTS}^{\circ+}$, segundo o método proposto por Larrauri, Rupérez e Saura-Calixto (1997). Foi utilizado uma curva padrão de Trolox (100 a $\left.2000 \mu \mathrm{mol} . \mathrm{L}^{-1}\right)$ e os resultados foram expressos como $\mu$ mol Trolox. $g^{-1}$ de amostra.

\subsubsection{Análise dos dados}

Os 16 ensaios compostos pelas diferentes misturas dos solventes extratores foram avaliados pelas seguintes funções respostas: $y_{1}=$ compostos fenólicos (mg EAG.100g $\left.{ }^{1}\right), y_{2}=$ Atividade antioxidante medida pelo método ABTS ( $\mu \mathrm{mol}$ de Trolox.g de amostra $\left.^{-1}\right), y_{3}=$ Atividade antioxidante medida pelo método DPPH $(\mu \mathrm{mol}$ de Trolox.g de amostra $^{-1}$ ). O modelo canônico de Scheffé (1963) (Equação 1) foi ajustado aos dados experimentais e testados os modelos linear, quadrático e cúbico para a obtenção dos respectivos coeficientes de regressão.

$$
y=\sum_{i=1}^{q} b_{i}^{*} x_{i}+\sum_{i<j}^{q} \sum_{j}^{q} b_{i j}^{*} x_{i} x_{j}+\sum_{i<j}^{q} \sum_{j<k}^{q} \sum_{k}^{q} b_{i j k}^{*} x_{i} x_{j} x_{k}+\cdots+b_{12 \ldots q}^{*} x_{1} x_{2} \ldots x_{q} \quad \text { (Equação 1) }
$$

Em que, $y=$ função da estimativa da resposta, $b_{i}{ }^{*}$ coeficientes estimados da regressão, $x_{i}=$ são os níveis codificados das dependentes, sendo $1>x_{i}>0$ e $\sum_{x i}=1,0$.

A qualidade do ajuste dos modelos aos dados experimentais foi verificada pela análise de variância (ANOVA) da regressão e coeficiente de determinação $\left(R^{2}\right)$. As repetições forneceram os graus de liberdade para obter o erro puro e conseqüentemente, a análise da falta de ajuste. Todos os cálculos e construções dos gráficos foram realizados utilizando o programa Statistica 10.0 (StatSoft Inc, 2011). A partir das funções respostas investigadas as combinações foram otimizadas a partir dos gráficos gerados da superfície de resposta e parâmetros de desejabilidade.

\section{Resultados e Discussão}

A partir dos valores médios das respectivas funções respostas (Tabela 1) dos 16 ensaios realizados e aplicação do planejamento experimental de misturas simplexcentroide (Figura 1), foram obtidos os modelos das equações $\hat{y}_{1}$ (teor de compostos fenólicos), $\hat{y}_{2}$ (atividade antioxidante pelo método ABTS), $\hat{y}_{3}$ (atividade antioxidante pelo método DPPH) com os seus respectivos coeficientes de regressão e análise de variância dos modelos matemáticos (Tabela 2). Os modelos matemáticos $\hat{y}_{1}, \hat{y}_{2}, \hat{y}_{3}$, apresentaram efeitos linear, quadrático e cúbicos significativos ao nível de $95 \%$ de probabilidade. As equações dos modelos matemáticos não apresentaram falta de ajuste significativa $(\notin 0,05)$ e os coeficientes de determinação $R^{2}$ (Tabela 2) foram respectivamente de $0,99,0,98$ e 0,97 . Portanto, estes modelos foram satisfatórios para explicar o efeito dos solventes água, acetona, etanol e metanol na extração dos compostos fenólicos e antioxidantes da polpa do abacate. 
Tabela 1. Coeficientes de regressão e análise de variância dos modelos matemáticos.

\begin{tabular}{llllllll}
\hline \multirow{2}{*}{ Ensaio } & \multicolumn{3}{c}{ Variáveis } & \multicolumn{3}{c}{ Funções-resposta* $^{*}$} \\
\cline { 2 - 8 } & $\mathrm{X}_{1}$ & $\mathrm{X}_{2}$ & $\mathrm{X}_{3}$ & $\mathrm{X}_{4}$ & $\mathrm{Y}_{1}$ & $\mathrm{Y}_{2}$ & $\mathrm{Y}_{3}$ \\
\hline 1 & 1,00 & 0,00 & 0,00 & 0,00 & 18,77 & 1,04 & 0,42 \\
2 & 0,00 & 1,00 & 0,00 & 0,00 & 23,73 & 2,01 & 0,74 \\
3 & 0,00 & 0,00 & 1,00 & 0,00 & 23,11 & 1,85 & 0,98 \\
4 & 0,00 & 0,00 & 0,00 & 1,00 & 19,01 & 2,07 & 1,19 \\
5 & 0,50 & 0,50 & 0,00 & 0,00 & 33,75 & 2,74 & 1,44 \\
6 & 0,50 & 0,00 & 0,50 & 0,00 & 27,56 & 2,00 & 0,82 \\
7 & 0,50 & 0,00 & 0,00 & 0,50 & 27,85 & 2,13 & 0,00 \\
8 & 0,00 & 0,50 & 0,50 & 0,00 & 20,48 & 1,94 & 1,05 \\
9 & 0,00 & 0,50 & 0,00 & 0,50 & 23,99 & 1,93 & 1,14 \\
10 & 0,00 & 0,00 & 0,50 & 0,50 & 25,54 & 2,05 & 1,15 \\
11 & 0,33 & 0,33 & 0,33 & 0,00 & 29,97 & 2,44 & 1,07 \\
12 & 0,33 & 0,33 & 0,00 & 0,33 & 28,46 & 2,27 & 1,26 \\
13 & 0,33 & 0,00 & 0,33 & 0,33 & 28,34 & 2,39 & 1,34 \\
14 & 0,00 & 0,33 & 0,33 & 0,33 & 21,66 & 1,99 & 1,08 \\
15 & 0,25 & 0,25 & 0,25 & 0,25 & 28,03 & 2,32 & 1,29 \\
16 & 0,25 & 0,25 & 0,25 & 0,25 & 28,30 & 2,44 & 1,24 \\
\hline
\end{tabular}

ajustados às variáveis resposta.

${ }^{*} X_{1}=$ Água, $X_{2}=$ Acetona, $X_{3}=$ Etanol e $X_{4}=$ Metanol. ${ }^{*} Y_{1}=$ Compostos fenólicos (mg EAG.100 g ${ }^{-1}$ ), $Y_{2}=$ Capacidade antioxidante pelo método ABTS ( $\mu$ mol de Trolox/g de amostra) e $Y_{3}=$ Capacidade antioxidante pelo método DPPH ( $\mu \mathrm{mol}$ de Trolox/g de amostra).

Tabela 2. Coeficientes de regressão e análise de variância dos modelos matemáticos ajustados às variáveis resposta.

\begin{tabular}{|c|c|c|c|}
\hline \multirow[b]{2}{*}{ Coeficientes } & \multicolumn{3}{|c|}{ Variáveis Resposta* } \\
\hline & $y_{1}$ & $y_{2}$ & $y_{3}$ \\
\hline \multicolumn{4}{|l|}{ Linear } \\
\hline$\beta_{1}$ & $18,78^{\star}$ & $1,04^{*}$ & $0,45^{\star}$ \\
\hline$\beta_{2}$ & $23,73^{\star}$ & $1,97^{\star}$ & $0,79 *$ \\
\hline$\beta_{3}$ & $23,12^{*}$ & $1,89 *$ & $1,08 *$ \\
\hline$\beta_{4}$ & $19,02^{*}$ & $2,06^{\star}$ & $1,19 *$ \\
\hline \multicolumn{4}{|l|}{ Quadrático } \\
\hline$\beta_{12}$ & $49,59 *$ & $4,90^{\star}$ & $3,33^{*}$ \\
\hline$\beta_{13}$ & $26,05^{\star}$ & $2,13^{\star}$ & - \\
\hline$\beta_{14}$ & $35,43^{*}$ & $2,28^{\star}$ & $-3,24^{*}$ \\
\hline$\beta_{23}$ & $-12,16^{\star}$ & - & - \\
\hline$\beta_{24}$ & $10,06^{*}$ & - & 0,63 \\
\hline$\beta_{34}$ & $17,49 *$ & - & - \\
\hline \multicolumn{4}{|l|}{ Cúbico } \\
\hline$\beta_{123}$ & $36,70^{\star}$ & 1,14 & $-2,95$ \\
\hline$\beta_{124}$ & - & $-5,64^{*}$ & $8,80 *$ \\
\hline$\beta_{134}$ & - & $6,83^{\star}$ & $20,21^{*}$ \\
\hline$\beta_{234}$ & - & - & $-1,49$ \\
\hline $\mathrm{R}^{2}$ & 0,99 & 0,98 & 0,97 \\
\hline Falta de ajuste (p) & 0,14 & 0,80 & 0,28 \\
\hline
\end{tabular}

${ }^{*} \mathrm{y}_{1}=$ Compostos fenólicos (mg EAG.100 $\mathrm{g}^{-1}$ ), $\mathrm{y}_{2}=$ Capacidade antioxidante pelo método ABTS ( $\mu \mathrm{mol}$ de Trolox. $\mathrm{g}^{-1}$ de amostra) e $\mathrm{y}_{3}=$ Capacidade antioxidante pelo método DPPH ( $\mu \mathrm{mol}$ de Trolox. $g^{-1}$ de amostra). 
Para a função-resposta compostos fenólicos $\left(\hat{y}_{1}\right)$, foram considerados os termos lineares, quadráticos e cúbicos significativos. O teor de compostos fenólicos na polpa de abacate variou de 18,77 a 33,75 mg EAG.100 ${ }^{-1}$ (Tabela 1). Pelos coeficientes da equação $\left(\hat{y}_{1}\right)$ as variáveis $X_{2}$ (acetona) e $X_{3}$ (etanol) apresentaram maior contribuição quando utilizadas sem a combinação com outro solvente, sendo que o efeito das variáveis foi potencializado quando utilizada a combinação das variáveis $X_{1}$ (água) e $X_{2}$ (acetona), o que pode ser confirmado pelo valor elevado do coeficiente $\beta_{12}(49,59)$ e pelo valor de compostos fenólicos obtidos no ensaio 5 (Tabela 1) (33,75 mg EAG. $\left.100 \mathrm{~g}^{-1}\right)$. Isto indica a importância de utilizar a ferramenta de superfície de resposta na otimização da extração, uma vez que é possível investigar toda a região experimental e as diferentes combinações de solventes, com um número reduzido de experimentos. Analisando a superfície de resposta (Figura 2a) observa-se que há uma tendência de aumentar o teor de compostos fenólicos com o aumento na proporção de acetona e água utilizados na mistura de solventes extratores. Em estudo realizado por Soong e Barlow (2004), foi obtido teor de 1,3 mg EAG. $\mathrm{g}^{-1}$ para amostras de abacate, de outra variedade, utilizando os solventes extratores água (50\%) e etanol (50\%). Wang, Bostic e Gu (2010) analisaram polpa de abacates de diferentes variedades, cujos compostos fenólicos foram extraídos por uma mistura de água, acetona e ácido acético, e obtiveram valores que variaram de 0,6 a 4,9 EAG. $\mathrm{g}^{-1}$, sendo que valores mais elevados de compostos fenólicos foram obtidos na semente (até 51,6 EAG. $\mathrm{g}^{-1}$ ) e na casca $\left(13,9\right.$ EAG. $\left.g^{-1}\right)$. A composição fenólica dos frutos, como o abacate, é determinada por fatores genéticos e ambientais, e pode ser modificada por reações oxidativas que ocorrem durante a pós-colheita, resultado do processamento e/ou estocagem (TOMÁS-BARBERÁN; ESPÍN, 2001), o que pode explicar os resultados distintos obtidos neste trabalho em relação aos dados da literatura. Além disso, muitos estudos têm mostrado que os compostos fenólicos geralmente diminuem em frutos climatérios durante o amadurecimento, como consequência das condições de armazenamento (HAARD; CHISM, 1996; MITRA; BALDWIN, 1997).
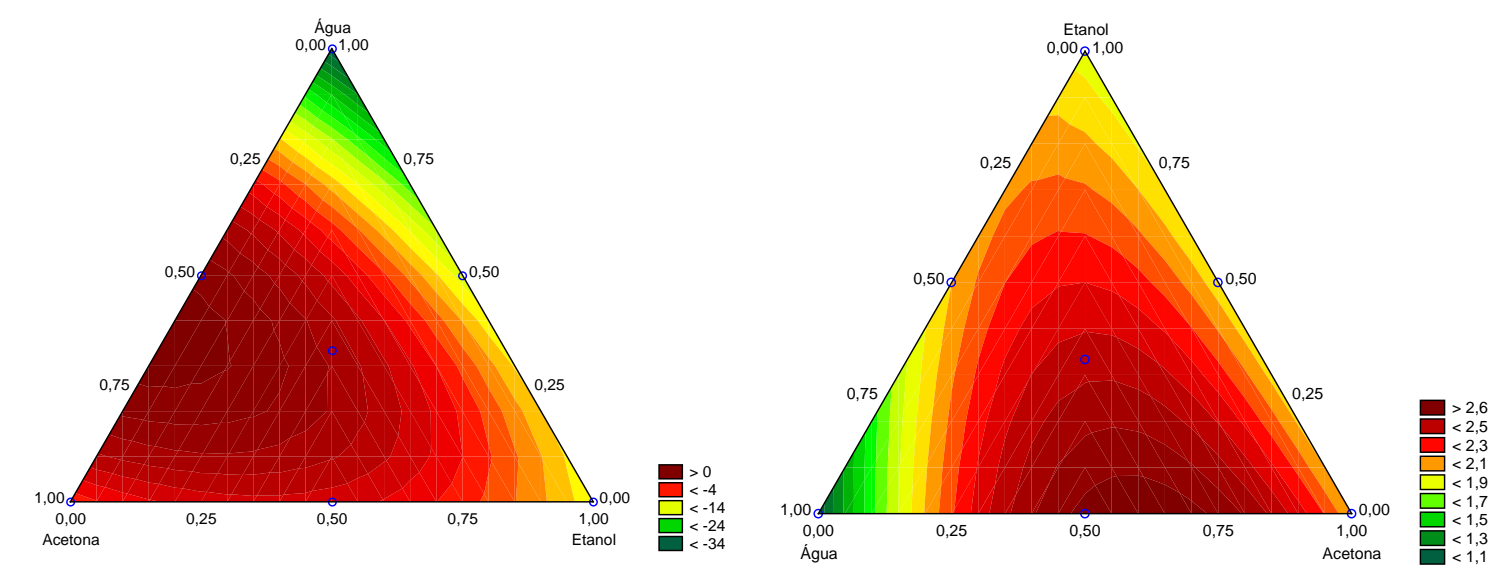


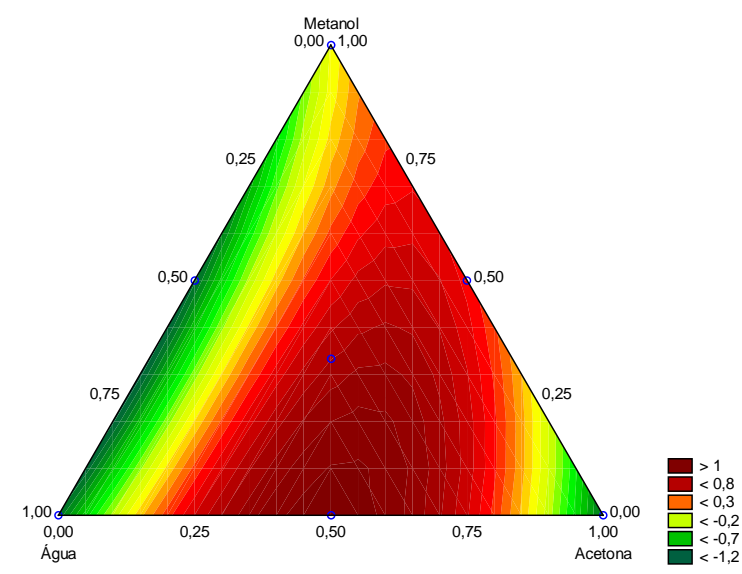

C

Figura 2. Superfície de resposta para $y_{1}=$ Compostos fenólicos (mg EAG.100g $\left.{ }^{-1}\right)(a)$, $\mathrm{y}_{2}=$ Capacidade antioxidante pelo método ABTS ( $\mu$ mol de Trolox.g de amostra ${ }^{-1}$ ) (b) e $\mathrm{y}_{3}=$ Capacidade antioxidante pelo método DPPH ( $\mu$ mol de Trolox.g de amostra ${ }^{-1}$ ) (c) para misturas dos solventes água, acetona, etanol e metanol.

A capacidade antioxidante quantificada pelo método ABTS na polpa de abacate variou de 1,04 a 2,74 $\mu \mathrm{mol}$ de Trolox. $\mathrm{g}^{-1}$ de amostra (Tabela 1). Para as diferentes misturas dos solventes extratores água, acetona, etanol e metanol a função-resposta $y_{2}$ (atividade antioxidante pelo método ABTS) apresentou os termos lineares, quadráticos e cúbicos significativos, sendo que um dos termos cúbico não foi significativo, mas foi mantido na equação devido a sua contribuição no ajuste do modelo matemático (Tabela 2). A variável $\mathrm{X}_{4}$ (metanol) apresentou mais expressiva contribuição quando utilizada como solvente único na extração, porém, maior contribuição foi observada quando se utilizou as variáveis $X_{1}$ (água) e $X_{2}$ (acetona) em combinação (ensaio 5) (Tabela 1). Analisando a superfície de resposta (Figura 2b) observa-se que também há uma tendência de aumentar a atividade antioxidante medida pelo método ABTS com o aumento na proporção de acetona e água utilizados na mistura de solventes extratores. Menor atividade antioxidante medida pelo método ABTS pode ser observada quando foi utilizado apenas água $\left(X_{1}\right)$ como solvente extrator (Figura 2b). Em estudo realizado por Soong e Barlow (2004), foi obtido 4,9 $\mu \mathrm{mol} . \mathrm{g}^{-1}$ para amostras de abacate, de outra variedade, utilizando os solventes extratores água (50\%) e etanol (50\%), portanto, valores superiores aos encontrados neste estudo com o mesmo par de solventes $\left(2,0 \mu \mathrm{mol} . \mathrm{g}^{-1}\right)$.

A capacidade antioxidante quantificada pelo método DPPH na polpa de abacate variou de 0,00 a 1,44 $\mu \mathrm{mol}$ de Trolox. $\mathrm{g}^{-1}$ de amostra (Tabela 1). Para as diferentes misturas dos solventes extratores água, acetona, etanol e metanol a função-resposta $y_{3}$ (atividade antioxidante pelo método DPPH) apresentou os termos lineares, quadráticos e cúbicos significativos, sendo que alguns termos quadráticos e cúbicos não foram significativos, mas foram mantidos na equação devido a sua contribuição no ajuste do modelo matemático (Tabela 2). A variável $\mathrm{X}_{4}$ (metanol) apresentou contribuição mais relevante quando utilizada como solvente único na extração, assim como ocorreu na função resposta $y_{2}$ (atividade antioxidante pelo método ABTS). Maior contribuição foi observada quando se utilizou as variáveis $X_{1}$ (água) e $X_{2}$ (acetona) em combinação (ensaio 5) (Tabela 1) e menor contribuição foi observada para a combinação das variáveis $\mathrm{X}_{1}$ (água) e $\mathrm{X}_{4}$ (metanol), indicando que a mistura destes solventes diminui a 
extração dos compostos antioxidantes quantificados pelo método DPPH. Ainda, observando os coeficientes do modelo obtido, pode-se observar que maiores teores de compostos antioxidantes são recuperados utilizando uma mistura de solventes $X_{1}$ (água), $X_{2}$ (acetona) e $X_{4}$ (metanol) ou $X_{1}$ (água), $X_{3}$ (etanol) e $X_{4}$ (metanol). Analisando a superfície de resposta (Figura $2 c$ ) observa-se que há uma tendência de aumentar a atividade antioxidante medida pelo método DPPH com o aumento na proporção de acetona e água utilizados na mistura de solventes extratores, e que valores elevados de atividade antioxidante também podem ser obtidos com a mistura acetona $\left(X_{2}\right)$ e metanol $\left(X_{4}\right)$ e com a utilização dos demais solventes. Menor atividade antioxidante medida pelo método DPPH foi observada quando foram utilizadas apenas água $\left(\mathrm{X}_{1}\right)$ ou acetona $\left(\mathrm{X}_{2}\right)$ como solventes extratores (Figura $2 \mathrm{c}$ ). Wang, Bostic e Gu (2010) analisaram polpa de abacates de diferentes variedades, sendo os compostos antioxidantes extraídos por uma mistura de água, acetona e ácido acético; os autores obtiveram valores de atividade antioxidante, quantificado pelo método $\mathrm{DPPH}$, de 0,4 a $1,4 \mu \mathrm{mol}$ de Trolox. $\mathrm{g}^{-1}$ de amostra, sendo que valores mais elevados de antioxidantes foram obtidos na semente (até $240,2 \mu \mathrm{mol}$ de Trolox. $\left.{ }^{-1}\right)$ e na casca $(189,8 \mu \mathrm{mol}$ de Trolox. $\left.\mathrm{g}^{-1}\right)$. Apesar do conteúdo de compostos fenólicos totais não necessariamente estar envolvido na quantificação da atividade antioxidante, os resultados obtidos neste estudo apresentaram a mesma tendência, indicando a efetividade de utilizar um método de extração aplicável paralelamente à análise de compostos fenólicos e atividade antioxidante.

Um grande problema na área de alimentos é a multiplicidade de funções-respostas em um planejamento experimental que se deseja otimizar. Quando várias funçõesrespostas estão envolvidas, como no caso deste estudo, emprega-se o termo otimização de respostas combinadas ou desejabilidade para determinar a mistura que proporcione as melhores propriedades. O gráfico da desejabilidade (Figura 3) apresenta as proporções ótimas dos solventes água, acetona, etanol e metanol para extração de compostos fenólicos e compostos antioxidantes quantificados pelos métodos ABTS e DPPH da polpa do abacate.

Como estas quantificações são realizadas, geralmente, a partir de um único extrato do alimento, é necessário encontrar uma condição que seja ótima para todas as funçõesrespostas avaliadas. Neste sentido, foi possível prever uma região ótima de mistura dos solventes para extração dos compostos de interesse partir da análise das funções resposta estimadas $\hat{y}_{1}, \hat{y}_{2}, \hat{y}_{3}$ (Tabelas 1 e 2), superfície de resposta (Figura 2) e parâmetros de desejabilidade (Figura 3). A desejabilidade global da mistura de solventes com maior poder extrator (Figura 3) ocorreu quando $x_{1}=0,5, x_{2}=0,5$ e $x_{3}=0$ e $\mathrm{x}_{4}=0$, que corresponde a condição de $50 \%$ de água, $50 \%$ de acetona, $0 \%$ de etanol e $0 \%$ de metanol, que coincidiu com o ensaio 5 (Tabela 1 ) e, portanto, não houve a necessidade de validar o modelo proposto. 


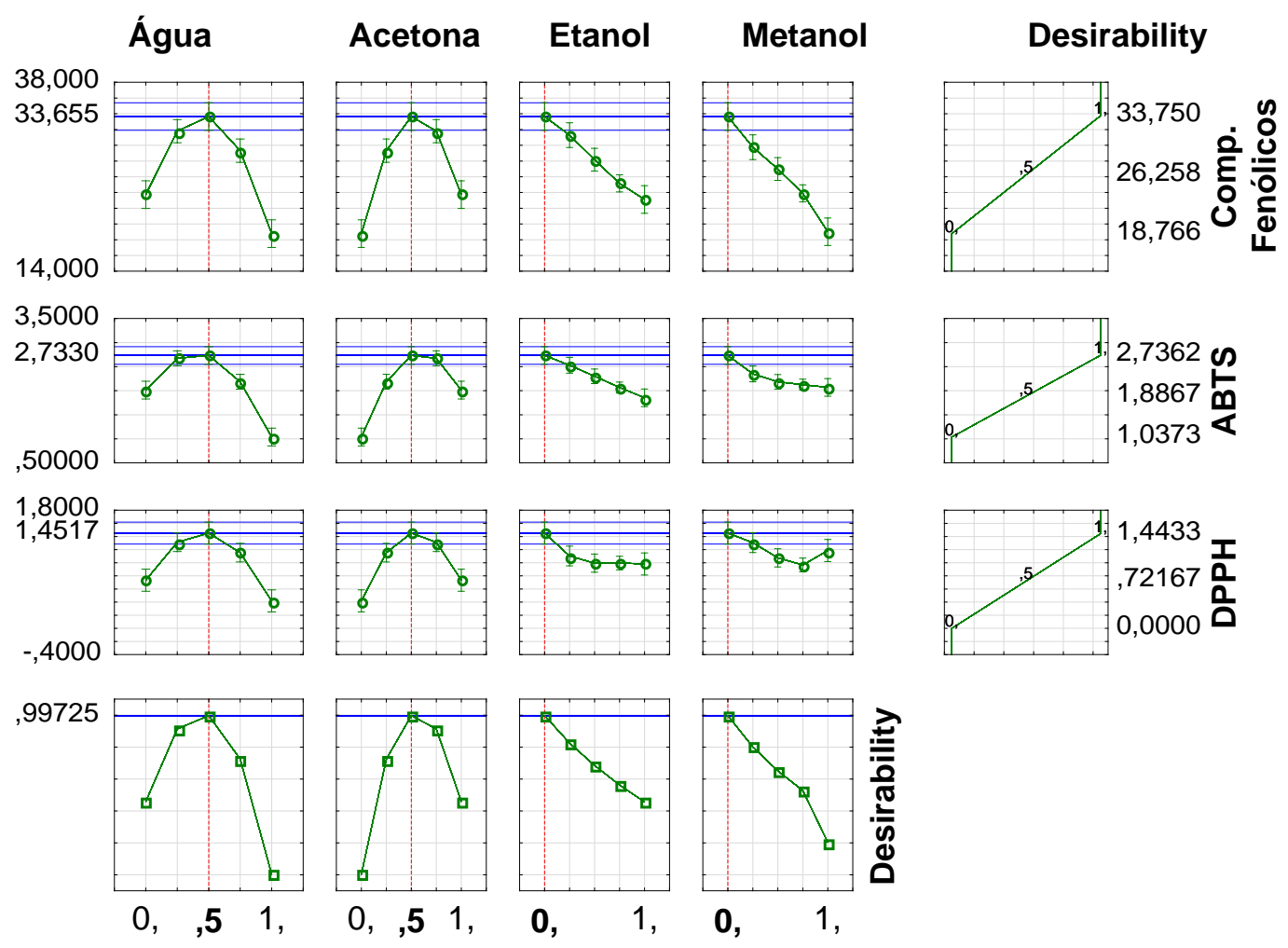

Figura 3. Proporções ótimas dos solventes: água, acetona, etanol e metanol para a extração de compostos fenólicos $\left(\mathrm{y}_{1}\right)$, capacidade antioxidante por ABTS $\left(\mathrm{y}_{2}\right)$ e capacidade antioxidante por DPPH da polpa do abacate utilizando o parâmetro de desejabilidade.

\section{Conclusão}

O planejamento experimental para misturas do tipo simplex-centroide demonstrou ser aplicável e de grande utilidade para avaliação de compostos bioativos em polpa de abacate. A partir da otimização de respostas combinadas foi possível determinar a combinação de solventes mais efetiva para extração de compostos fenólicos e antioxidantes da polpa de abacate variedade Quintal. A mistura de $50 \%$ de água e $50 \%$ de acetona revelou-se a mais apropriada para os propósitos avaliados.

\section{Referências}

ADA. American Dietetic Association. Position of The American Dietetic Association: Functional foods. Journal of the American Dietetic Association, v.99, p.1278-1285, 1999. https://doi.org/10.1016/S0002-8223(99)00314-4.

BRAND-WILLIAMS, W.; CUVELIER, M. E.; BERSET, C. Use of a free radical method to evaluate antioxidant activity. Lebensmittel-Wissenschaft Technologie, v.28, p.2530, 1995. https://doi.org/10.1016/S0023-6438(95)80008-5.

GEISSMAN, T. A.; DITTMAR, H. F. K. A proanthocyanidin from avocado seed. Phytochemistry, v.4, p.359-368, 1965. https://doi.org/10.1016/S0031-9422(00)861889. 
GÓMEZ-LÓPEZ, V. M. Characterization of avocado (Persea americana Mill.) varieties of very low oil content. Journal of Agricultural and Food Chemistry, v.46, p.36433647, 1998. https://doi.org/10.1021/jf9706890.

JACOBO-VELÁZQUEZ, D.A.; HERNÁNDEZ-BRENES, C. Stability of avocado paste carotenoids as affected by high hydrostatic pressure processing and storage. Innovative Food Science \& Emerging Technologies, v.16, p.121-128, 2012. https://doi.org/10.1016/j.ifset.2012.05.001.

KEVERS, C. et al. Evolution of antioxidant capacity during storage of selected fruits and vegetables. Journal of Agricultural and Food Chemistry, v.55, n.21, p.85968603, 2007. https://doi.org/10.1021/jf071736j.

HAARD, N. F.; CHISM, G. W. Characteristics of edible plant tissues. In: DAMODARAN, S. Fennema's food chemistry. New York: Marcel Dekker, 1996, p.943-1011.

LARRAURI, J.A.; RUPÉREZ, P.; SAURA-CALIXTO, F. Effect of drying temperature on the stability of polyphenols and antioxidant activity of red grape pomace peels. Journal of Agricultural and Food Chemistry v. 45, p.1390-1393, 1997. https://doi.org/10.1021/jf960282f.

LEE, J.; KOO, N.; MIN, D. Reactive oxygen species, aging, and antioxidative nutraceuticals. Comprehensive Reviews in Food Science and Food Safety, v.3. n.1, p.21-33, 2004. https://doi.org/10.1111/j.1541-4337.2004.tb00058.x.

LU, Q.Y. et al. Inhibition of prostate cancer cell growth by an avocado extract: Role of lipid-soluble bioactive substances. The Journal of Nutritional Biochemistry, v.16, p.23-30, 2005. https://doi.org/10.1016/j.jnutbio.2004.08.003.

LUTHRIA, D. L. Significance of sample preparation in developing analytical methodologies for accurate estimation of bioactive com-pounds in functional foods. Journal of Science of Food and Agriculture, v.86, p.2266-2272, 2006. https://doi.org/10.1002/jsfa.2666.

LUTHRIA, D.L. Influence of experimental conditions on the extraction of phenolic compounds from parsley (Petroselinum crispum) flakes using a pressurized liquid $\begin{array}{lllll}\text { extractor. Food } \quad \text { Chemistry, } & \text { v.107, }\end{array}$ https://doi.org/10.1016/j.foodchem.2007.08.074.

MITRA, S. K.; BALDWIN, E. A. Mango. In: MITRA, S. K. (Ed.). Postharvest physiology storage of tropical and subtropical fruit. New York: CAB Internacional, 1997. $431 \mathrm{p}$

PEREIRA, M.C. et al. Characterization, bioactive compounds and antioxidant potential of three Brazilian fruits. Journal of Food Composition and Analysis, v.29, p.19-24, 2013. http://dx.doi.org/10.1016/j.jfca.2012.07.013.

POMPEU, D. R.; SILVA, E. M.; ROGEZ, H. Optimization of the solvent extraction of phenolic antioxidants from fruits of Euterpe oleracea using Response Surface Methodology. Bioresource Technology, v.100, p.6076-6082, 2009. https://doi.org/10.1016/j.biortech.2009.03.083.

RAMARATHNAM, N. et al. The contribution of plant food antioxidants to human health. Trends in Food Science and Technology, v.6, p.75-82, 1995. https://doi.org/10.1016/S0924-2244(00)88967-0. 
ROBBINS, R. J. Phenolic acids in foods: An overview of analytical methodology. Journal of Agriculture and Food Chemistry, v.51, p.2866-2887, 2003. https://doi.org/10.1021/jf026182t.

SAMPAIO, A. C.; LEONEL, S. Abacate: aspectos técnicos da produção. Universidade Estadual Paulista: Cultura Acadêmica Editora, São Paulo, SP, 2008.

SCHEFFÉ, H. The simplex-centroid design for experiments with mixtures. Journal of the royal statistical society. Series B (Methodological), 25, n.2, p.235-263, 1963. https://doi.org/10.1111/j.2517-6161.1963.tb00506.x.

SOONG, Y.Y.; BARLOW, P.J. Antioxidant activity and phenolic content of selected fruit $\begin{array}{llll}\text { seeds. Food } \quad \text { Chemistry, } & \text { v.88, }\end{array}$ https://doi.org/10.1016/j.foodchem.2004.02.003.

SPIGNO, G.; TRAMELLI, L.; FAVERI, D.M. Effects of extraction time, temperature and solvent on concentration and antioxidant activity of grape marc phenolics. Journal of $\begin{array}{llll}\text { Food } \quad \text { Engineering, } & \text { v.81, } 2007 .\end{array}$ https://doi.org/10.1016/j.jfoodeng.2006.10.021.

SWAIN, T.; HILLIS, W. T. The phenolic constituents of Prunnus domestica. Journal of $\begin{array}{lllll}\text { Science and Food Agriculture, v.10, p.135-144, } 1959 . & \end{array}$ https://doi.org/10.1002/jsfa.2740100110.

TOMÁS-BARBERÁN, F.; ESPÍN, J. C. Phenolic compounds and related enzymes as determinants of quality in fruits and vegetables. Journal of the Science of Food and Agriculture, v. 81, p. 853- 876, 2001. https://doi.org/10.1002/jsfa.885.

WANG, W.; BOSTIC, T.R.; GU, L. Antioxidant capacities, procyanidins and pigments in avocados of different strains and cultivars. Food Chemistry, v.122, n.4, p.1193-1198, 2010. https://doi.org/10.1016/j.foodchem.2010.03.114.

\section{Autores}

Tahís Regina Baú ${ }^{1}$, Carla Rosane Barboza Mendonça ${ }^{2, *}$

1. Curso de Pós-Graduação Lato Sensu em Ciência dos Alimentos, Centro de Ciências Químicas, Farmacêuticas e de Alimentos, Universidade Federal de Pelotas, Campus Capão do Leão, Caixa Postal 354, CEP. 96010-900, Pelotas, RS, Brasil; Instituto Federal de Santa Catarina, CEP 89900-000, São Miguel do Oeste/SC, Brasil

2. Centro de Ciências Químicas, Farmacêuticas e de Alimentos, Universidade Federal de Pelotas, Campus Capão do Leão, Caixa Postal 354, CEP. 96010-900, Pelotas, RS, Brasil

*Autor para correspondência: carlaufpel@hotmail.com 